\title{
Autonomous, Agile Micro- Satellites, and Supporting Technologies
}

A. G. Ledebuhr, J. F. Kordas, L. C. Ng, M. S. Jones, J. C. Whitehead, E. Brietfeller, R. J. Gaughan, M. D. Dittman, B. Wilson

This article was submitted to

AIAA Space Technology Conference and Exposition Albuquerque, New Mexico

September 28-30, 1999

U.S. Department of Energy

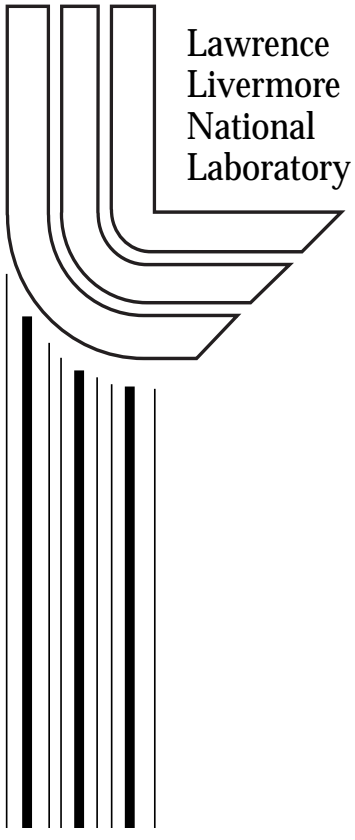

July 19, 1999 


\section{DISCLAIMER}

This document was prepared as an account of work sponsored by an agency of the United States Government. Neither the United States Government nor the University of California nor any of their employees, makes any warranty, express or implied, or assumes any legal liability or responsibility for the accuracy, completeness, or usefulness of any information, apparatus, product, or process disclosed, or represents that its use would not infringe privately owned rights. Reference herein to any specific commercial product, process, or service by trade name, trademark, manufacturer, or otherwise, does not necessarily constitute or imply its endorsement, recommendation, or favoring by the United States Government or the University of California. The views and opinions of authors expressed herein do not necessarily state or reflect those of the United States Government or the University of California, and shall not be used for advertising or product endorsement purposes.

This is a preprint of a paper intended for publication in a journal or proceedings. Since changes may be made before publication, this preprint is made available with the understanding that it will not be cited or reproduced without the permission of the author.

This report has been reproduced directly from the best available copy.

Available to DOE and DOE contractors from the

Office of Scientific and Technical Information

P.O. Box 62, Oak Ridge, TN 37831

Prices available from (423) 576-8401

http:/ / apollo.osti.gov/bridge/

Available to the public from the National Technical Information Service

U.S. Department of Commerce 5285 Port Royal Rd., Springfield, VA 22161 http://www.ntis.gov/

OR

Lawrence Livermore National Laboratory Technical Information Department's Digital Library http://www.llnl.gov/tid/Library.html 


\title{
AUTONOMOUS, AGILE MICRO-SATELLITES, AND SUPPORTING TECHNOLOGIES
}

\author{
A. G. Ledebuhr, J. F. Kordas, L. C. Ng, M. S. Jones, \\ J. C. Whitehead, E. Breitfeller, R. J. Gaughan, M. D. Dittman, and B. A. Wilson \\ Lawrence Livermore National Laboratory \\ P.O. Box 808 , L-043 \\ Livermore, CA 94550 \\ (925) 423-1184 \\ ledebuhr1@1lnl.gov
}

\begin{abstract}
This paper updates the on-going effort at Lawrence Livermore National Laboratory to develop autonomous, agile micro-satellites (MicroSats). The objective of this development effort is to develop MicroSats weighing only a few tens of kilograms, that are able to autonomously perform precision maneuvers and can be used telerobotically in a variety of mission modes. The required capabilities include satellite rendezvous, inspection, proximity-operations, docking, and servicing. The MicroSat carries an integrated proximity-operations sensor-suite incorporating advanced avionics. A new self-pressurizing propulsion system utilizing a miniaturized pump and non-toxic mono-propellant hydrogen peroxide was successfully tested. This system can provide a nominal $25 \mathrm{~kg}$ MicroSat with $200-300 \mathrm{~m} / \mathrm{s}$ delta-v including a warm-gas attitude control system. The avionics is based on the latest PowerPC processor using a CompactPCI bus architecture, which is modular, high-performance and processor-independent. This leverages commercial-off-the-shelf (COTS) technologies and minimizes the effects of future changes in processors. The MicroSat software development environment uses the VxWorks real-time operating system (RTOS) that provides a rapid development environment for integration of new software modules, allowing early integration and test. We will summarize results of recent integrated ground flight testing of our latest non-toxic pumped propulsion MicroSat testbed vehicle operated on our unique dynamic air-rail.
\end{abstract}

\section{Introduction}

This paper updates the on-going effort at Lawrence Livermore National Laboratory (LLNL) to develop autonomous, agile micro-satellites (or MicroSats) capable of performing precision maneuvers in space. ${ }^{1}$ It summarizes the latest advances in the propulsion, sensor, and avionics areas. Results of recent integrated ground flight testing of our latest non-toxic pumped propulsion MicroSat testbed vehicle are reported. The objective of this development effort is to develop MicroSats weighing only a few tens of kilograms, with 1 to $2 \mathrm{~km} / \mathrm{s}$ of $\Delta \mathrm{v}$, capable of performing precision maneuvers autonomously or telerobotically in space.

\section{Potential Missions}

Potential missions for MicroSats center on space "logistics" missions such as rescue and servicing, that will require vehicles with the ability to perform a variety of functions autonomously or semi-autonomously. These include rendezvous, inspection, proximity-operations (formation flying), docking, and robotic servicing functions (refueling, repowering or repairing). Figure 1 shows the various MicroSat missions of interest. Each of these mission functions require key technical capabilities. For example, rendezvous with a space asset by performing orbit matching requires precision maneuvering. Inspection of a space asset by flying to different view points of an inspection geometry requires precision MicroSat positioning, pointing, tracking and imaging. A satellite rescue might involve docking, repairing or refueling the satellite, followed by a departure, and post-rescue inspection. The rescue mission requires precision guidance, navigation, and control; precision ranging; high resolution imaging; and some type of micro-robotic manipulation. For example, a variety of robotic arms could be used to enable the MicroSat to perform a physical dock with a target satellite. Figure 2 illustrates one such approach. Here a MicroSat deploys four mechanical arms to grapple the launch vehicle interface flange as it lands on the target satellite. Once docked, a precision 6 degrees-of-freedom manipulator (actuator) could be used to align and plug an external connector into a targeted satellite's umbilical connector for data collection, diagnostic measurements or re-powering. This servicing operation could be performed telerobotically from the ground, to provide the flexibility and problem solving of a human presence. Other space logistic operations such as the collection and de-orbiting of hazardous space debris (junk) require precision vehicle guidance, navigation and control and a precision homing strategy. 
Rendezvous

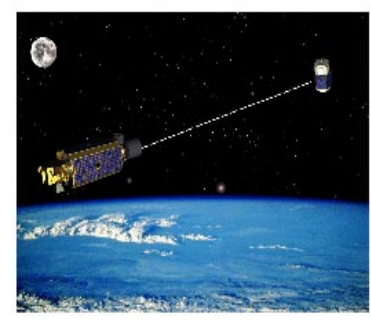

Docking

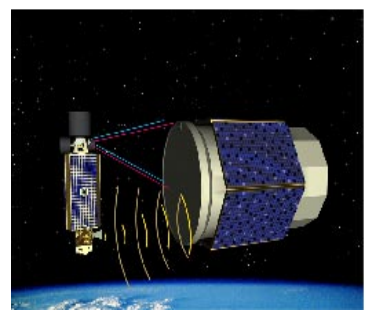

Inspection

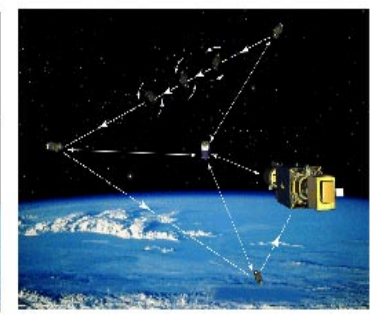

Servicing

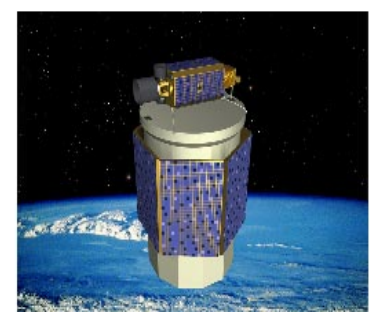

Proximity-Operations

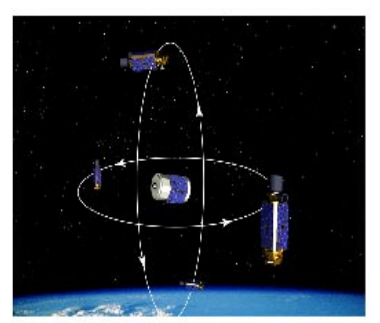

Performance Verification

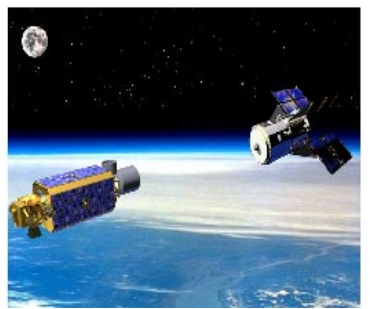

Figure 1 Potential missions of Micro-Satellites in Low Earth Orbit.

Formation flying, flying in concert with a space object or another MicroSat, requires station keeping, positioning, and precision state vector estimation.

Close-up inspection missions offer a means to remotely determine a satellite's health and status, and can collect data that can not be obtained from the ground. For example, a laser vibration sensor can determine bearing wear on moving components like momentum wheels, controlmoment gyros or solar array drives. Infrared sensing can observe thermal nonuniformities and detect leaks and differences in thermal insulation. Other missions may involve physically moving or towing a space object to a different orbit, constructing a 3D surface image of the object using stereo vision, estimating the object mass properties, and perhaps even reconstructing the internal structure of the object from 3D computed tomography. There are many potential missions that will become apparent once the basic system capability becomes routinely available.

A previous study ${ }^{1}$ has shown that a MicroSat with $300 \mathrm{~m} / \mathrm{s}$ of velocity change (or $\Delta \mathrm{v}$ ) is about the minimum necessary to carry out a basic mission, assuming the MicroSat is placed in the same orbit as the satellite to be inspected. Clearly vehicles with larger $\Delta \mathrm{v}$ offer multiple mission capabilities and the ability to change orbits. In addition if a spaceborne refueling capability is developed, then the mission utility of the MicroSat can be greatly extended.

In order to demonstrate many of these proximity operation capabilities, LLNL, under the sponsorship of the Air Force
Research Laboratory, has developed several MicroSat prototype vehicles for ground testing using a number of state-of-the-art technologies to support future Air Force missions. One mission concept calls for the MicroSat to demonstrate proximity-operations near a space object including the capability of soft docking. A proposed mission scenario is shown in Figure 3. An agile MicroSat will eject from the carrier vehicle to a distance of about $10 \mathrm{~m}$ and conduct a series of proximity operations within the $10 \mathrm{~m}$ radius sphere. For example, proximity inspection sequence via points at 1-2-3-4-5-6; circular stereo imaging via circles 6-3-1-5-6 and 6-4-1-2-6; soft landing via points 6-7-8-9; and formation flying at points $1,4,6$, and 2 . The mission is completed when the MicroSat has successfully demonstrated repeated soft-dockings with the carrier vehicle.

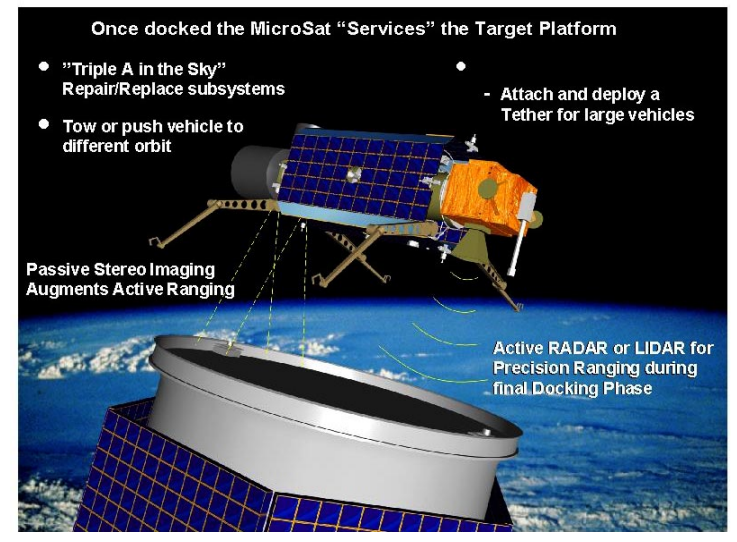

Figure 2 Artist conception of a MicroSat docking maneuver. 


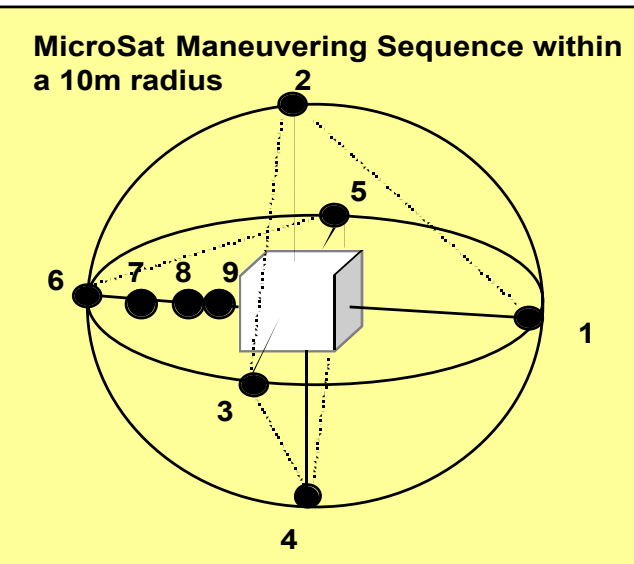

Proximity Maneuvers:

1. Octahedron imaging 1-2-3-4-5-6

2. Circular stereo imaging C63156-C64126

3. Soft docking 6-7-8-9

4. Formation flying $1,4,6,2$

Figure 3 A proposed proximity maneuver and soft docking mission scenario.

\section{Supporting Technologies}

\section{Propulsion}

A new self-pressurizing hydrogen peroxide propulsion system has been developed with liquid thrusters and gas jet attitude control without heavy gas storage vessels. A pump boosts the pressure of a small fraction of the hydrogen peroxide, so that reacted propellant can controllably pressurize its own source tank. The warm decomposition gas pressurizes the propellant tanks, powers the pump and is used by the attitude control jets for attitude control. Considering the single source reservoir, there is no need to

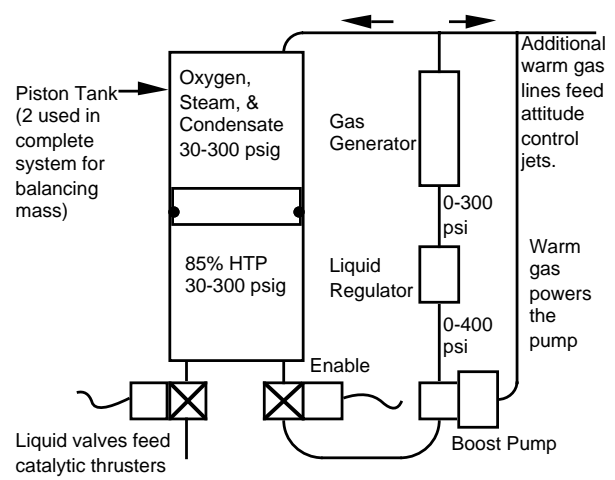

Figure 4 Schematic diagram of our first self-pressurizing propulsion system. apportion propellant between translational maneuvers and rotational control in advance.

Figure 4 shows a block diagram of the self-pressurizing propulsion subsystem. For operation, the liquid side of the tanks are filled with high test hydrogen peroxide (HTP) and the gas side charged with an initial charge of 50 psi of air. For system startup, the liquid solenoid valve is simply opened. Propellant at 50 psi flows through the pump, regulator, and gas generator. With 50 psi driving the pump, the liquid outlet is boosted to a higher pressure, and the positive feedback loop is controlled by the liquid regulator which is set to shut at $300 \mathrm{psi}$.

A series of tests were conducted with the self-pressurizing portion only of the propulsion system. During this test series, it became clear that part of the advantage of a nontoxic propellant is that system testing could proceed without rigorously testing individual components for safety reasons. Data obtained included the rate of pressure rise, the pump frequency (audio), and several key temperatures. While the warm gas lines and gas generator operated in the $500{ }^{\circ} \mathrm{F}$ to $1200^{\circ} \mathrm{F}$ range, the tank and pump block remained below $300^{\circ} \mathrm{F}$ which is well within the long-term capability of aluminum alloys. During a videotaped test series in September 1998, successful starts were achieved at 15, 30, and $50 \mathrm{psi}$. Times to reach $300 \mathrm{psi}$ were $30 \mathrm{~s}, 20 \mathrm{~s}$, and 10 $\mathrm{s}$, respectively.

Pump cycling averaged approximately $1 \mathrm{~Hz}$ during startup (e.g. 40 exhaust pulses heard during a $20 \mathrm{~s}$ period). It stroked very slowly at the lowest pressures, then gradually went faster and finally slowed down as the liquid regulator began to shut. The scarcity of pump exhaust pulses after startup indicated that leakage was minimal through the warm gas dynamic seals.

The pump displacement is $3 \mathrm{cc}$, or 5-6 cc estimated output per cycle. Thus roughly $100 \mathrm{cc}$ of $\mathrm{H}_{2} \mathrm{O}_{2}$ were pumped in order to pressurize the $\sim 2$ liter tank ullage to $300 \mathrm{psi}$. Note that this was most of the tank volume, as only a small propellant load was used for these tests. Assuming all the water condenses, pressurizing 2 liters to $300 \mathrm{psi}(2 \mathrm{MPa})$ at $250{ }^{\circ} \mathrm{F}\left(\sim 400^{\circ} \mathrm{K}\right)$ requires 1.2 gram moles of oxygen, or $\sim 40$ grams. This amount of oxygen is present in $\sim 100$ grams $(73 \mathrm{cc})$ of $85 \% \mathrm{H}_{2} \mathrm{O}_{2}$. This calculation roughly accounts for the startup propellant volume (note some is needed to drive the pump), and is consistent with the conclusion that essentially all the steam in the tank condensed.

The complete self-pressurizing propulsion system was assembled as shown in the line drawing in figure 5. Tests 
were planned for horizontal thrusting only, so the boost pump was connected in place of the upper thruster. The latter's valve remained and was actuated to initiate selfpressurization. The pump orientation causes any rising bubbles in the liquid manifolds to move upstream instead of naturally escaping. Thus, potential problems with gas pockets in microgravity would become evident during ground testing. Other parts were located for mass balancing, and to confine the hottest tubing runs in a small area around the aft tank. A normally open vent valve was included on the warm gas circuit so the system would safely shut down upon loss of electrical power during ground testing. The initial low-pressure nitrogen charge was also introduced here.

The propulsion system had 16 warm gas jets to provide independent fine control of both translational position and angular orientation. Available valves having high temperature elastomer seal pucks were fitted with conical nozzles and used for these gas jets. Figure 5 represents a $9.85 \mathrm{~kg}$ dry propulsion system. This included over $2 \mathrm{~kg}$ of heavyweight attitude jets and $1.6 \mathrm{~kg}$ of stainless steel fittings, so at least $3 \mathrm{~kg}$ could be trimmed.

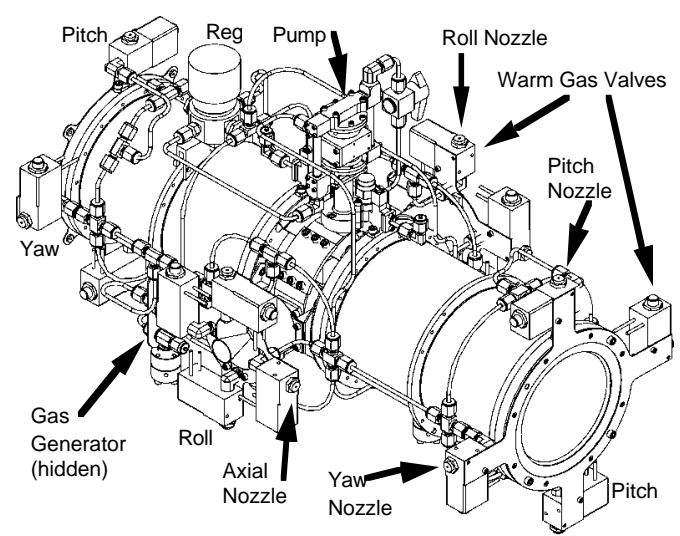

Figure 5. Complete self-pressurizing maneuvering system.

This system was incorporated into a prototype microsatellite for terrestrial maneuvering tests with the test results reported in a later section of this paper. For additional detail on the propulsion system see Reference 2 .

\section{Sensors}

The sensors are integrated into a single package that provides data for guidance, navigation, and control, and also provides images for transmission to the ground. A variety of sensor assemblies could be integrated into this package. The specific configuration presented here, as shown in figure 6, has two different visible imaging sensor systems, two laser rangers, a star tracker, and an IMU. The sensors complement each other, providing detailed information suitable for a variety of mission operations over distances from $10 \mathrm{~km}$ to docking contact. The visible cameras are based upon commercial CMOS active-pixel sensor (APS) imaging detectors. The laser rangers are modified commercially available systems, one for distant ranging and the other for proximity ranging. The star tracker is an upgrade of a wide-field system, originally developed and flown on the successful Clementine I Lunar mapping mission. The IMU is the commercially available space qualified LN200 by Litton.

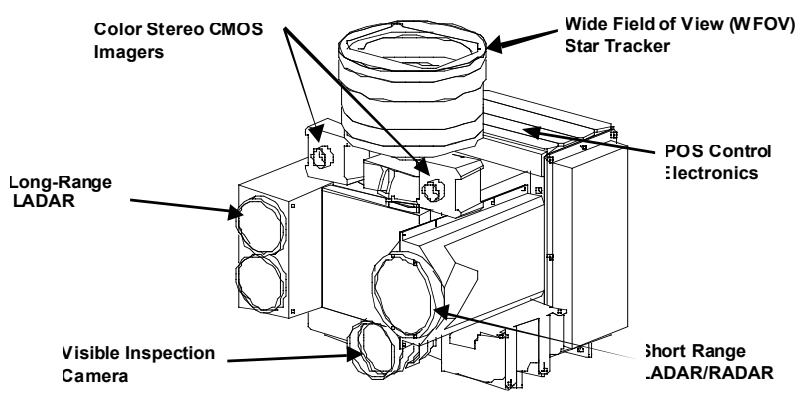

Figure 6 Multi-function integrated MicroSat Proximity Operations sensors head.

\section{Visible Imagers}

The Visible Inspection Camera has a $150 \mathrm{~mm}$ focal length lens imaging on a 1024 X 1024 CMOS APS array. A one meter target at a range of $5 \mathrm{~km}$ subtends three pixels on the detector. At $100 \mathrm{~m}$ the camera will resolve features as small as $7 \mathrm{~mm}$. The Visible Inspection Camera will continue to provide good imagery until handoff to the Color Stereo Pair at a range of 30 to 10 meters. The camera delivers digital data at a frame rate as high as $10 \mathrm{~Hz}$.

The Color Stereo Pair has two identical 28 degree FOV cameras using $640 \mathrm{X} 480$ pixel CMOS APS detectors that incorporate red, green and blue filters in a Bayer pattern. These also provide digital data at a frame rate up to $10 \mathrm{~Hz}$. At 10 meters each of the cameras can resolve features as small as $4 \mathrm{~mm}$ on the target satellite. The cameras are independent, and may be used individually (in monocular mode) or when used together (in stereo mode), can provide passive ranging information and also give a telepresence capability, for remote man-in-the-loop operation of the MicroSat.

CMOS APS detectors were chosen due to the large reductions in mass, volume, and power possible with these devices in comparison with standard CCDs. In addition to the savings for each single camera, there will be additional savings in the avionics because of the commonality of the camera interfaces. The CMOS sensors are extremely flexible and provide selectable, gain, frame rate, and integration times. In addition, they offer windowing or on- 
chip pixel summing allowing more rapid image acquisition or easier downlink of a region of interest.

\section{$\underline{\text { Rangers }}$}

There are two active laser rangers in the integrated sensor package. The long-range LADAR is a dual aperture timeof-flight system, using a modulated low-power infrared source. It's accurate to $2 \mathrm{~m}$ at $5 \mathrm{~km}$, and better than $1 \mathrm{~m}$ from $2 \mathrm{~km}$ to $<30 \mathrm{~m}$. The Proximity Ranger is a directfeedback system accurate to $<1 \mathrm{~cm}$ from $30 \mathrm{~m}$ to $0 \mathrm{~m}$ (Docking).

\section{$\underline{\text { IMU }}$}

The LN200 is currently being used in the ground test vehicles due to its performance and relatively low cost. This IMU has a measured drift rate of approximately $1 \%$ hour and is a ruggedized package suitable for use in space. There are a number of other IMUs that could be utilized in these vehicles including some new MEMs systems that currently promise reduced mass and power but at the expense of performance. Measured drift rates for MEMs IMUs are currently at the $10^{\circ}$ /hour rate, which if periodically updated with a Star Tracker is quite acceptable for MicroSat applications.

\section{WFOV Star Tracker}

A key sensor in the MicroSat is a wide-field-of-view (WFOV) Star Tracker that provides inertial orientation of the vehicle and updates for the IMU. The Star Tracker camera in conjunction with Stellar Compass software can provide a quaternion pointing accuracy of $450 \mu \mathrm{rad}, 3 \sigma$ in the roll axis, and $90 \mu \mathrm{rad}, 3 \sigma$ in pitch and yaw. The Star Tracker field of view is large enough to contain at least five bright stars $(\mathrm{Mv}=4.5)$ in any orientation. Single images are processed to identify unique stellar patterns and provide the determination of the inertial orientation of the MicroSat in real-time. The collection aperture of the lens is maximized for the greatest possible light gathering capability. At $\mathrm{F} / 1.25, \mathrm{~m}_{\mathrm{V}}=4.5 \mathrm{G} 0$ stars provide an integrated star signal that is 15 times the electronic noise from the focal plane. This level of signal gathering capability, matched with the wide field of view, ensures a $99.9 \%$ probability that 5 stars above minimum threshold will be available for the algorithm set for all possible quaternion pointing vectors. This allows the Star Tracker to handle the "lost in space" condition with a single star image frame and no other a priori knowledge of attitude. The latest Star Tracker generation replaces the previous CCD array with the same 1024 X 1024 pixel CMOS APS array used in the visible camera. The modified Star Tracker will have a 32 x 32 degree coverage using the existing lens design.

\section{Sensor Functions}

For a typical Inspection/Docking mission the sensors must support the various mission phases that the MicroSat must execute. In the initial Rendezvous phase, the sensors must first acquire the satellite to be inspected and then carry out the necessary Proximity-Operations during the Inspection and Docking Phases. During the Rendezvous phase, from $10 \mathrm{~km}$ to 10 meters, the visible imager will be required to identify the target and provide centroids to the guidance system to support an autonomous rendezvous operation. The Visible Imager provides the long-range acquisition of the target spacecraft and provides initial imagery of the target. At $5 \mathrm{~km}$, the distant ranger begins providing active range information with $\pm 2 \mathrm{~m}$ accuracy. At 100 meters the Inspection Phase begins. The visible imager will provide detailed images for downlink to the ground station. The $100 \mathrm{~m}$ to $10 \mathrm{~m}$ closing portion of the inspection phase continues to require input from the visible imager. A variable focus and magnification zoom lens system could provide flexibility and an overlap in capability beyond that possible with fixed focal length lenses. Technology trades are in process to determine the best flexible solution. At about 26 meters the proximity ranger begins to provide range information, with an accuracy of $\pm 1.5 \mathrm{~cm}$. At 10 meters the vehicle will switch to its pair of color inspection cameras, which will produce more detailed images for ground evaluation. The color stereo pair will again be required to provide information to the guidance and navigation control system during the $10 \mathrm{~m}$ to $10 \mathrm{~cm}$ closing portion of the Docking phase. For the docking maneuver the optical sensors will allow features to be identified, and in the terminal phase they will determine the precise location on the target where the hard dock will be made. The Stereo Color Inspection Camera pair produces close-in imagery of the target satellite. This system may be used in either stereoscopic or monoscopic mode. Stereo imagery provides passive range data for the GN\&C system.

The integrated sensor system described here supports the operational phases of a potential inspection/docking mission. However, the modular nature of the sensor components makes it relatively straightforward to modify or add to the sensor suite defined here to meet varying requirements of other specific missions. After docking operations are complete, the MicroSat will undock and depart the satellite. The sensor system now will produce new inspection images. 


\section{Avionics}

The MicroSat avionics system described here is in large part the same as that described previously ${ }^{1}$, but has been modified to accommodate a newer sensor suite and advances in COTS I/O modules. The avionics architecture is based on a high performance PowerPC processor and CompactPCI bus as shown in Figure 7. The PowerPC family is widely used in embedded systems for its performance and low power features. In addition, commercial versions of the PowerPC 603e have been tested, demonstrating significant inherent radiation tolerance. The CompactPCI bus is a high-performance, processor independent I/O bus, which provides an efficient path for processor upgrades. The system supports modern, real-time embedded software development environments. This design allows rapid code development, debugging, and testing. Its modular design leverages COTS technologies, permitting early integration and test of both hardware software elements. The chosen architecture provides a high performance solution for current and future MicroSat missions.

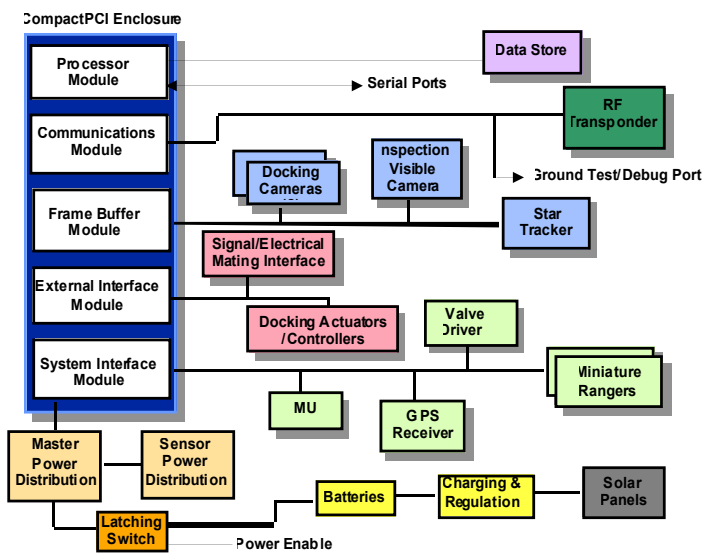

Figure 6 Avionics Architecture.

This same architecture and design approach, are being adopted by major aerospace system providers. Projects are now underway for developing radiation-hardened PowerPC CompactPCI modules.

\section{Processor Module}

As has been described in (1), the MicroSat processor module shown in Figure 8 contains a high-performance PowerPC 603e RISC CPU and utilizes a $33 \mathrm{MHz}, 32$ bit data path CompactPCI bus. The flight processor will be a COTS module with modifications for thermal management and radiation tolerant parts as needed.

The MicroSat local data store will be connected to the processor's controller port as indicated above. This will be a commercially available Flash Disk that has built-in error correction capability. The size of the disk selected will be based on the specifics of the mission profile. During a mission sequence the MicroSat will store on board the majority of imagery data collected for later down-link to

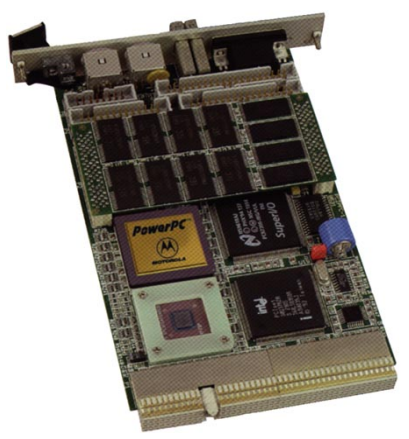

Figure 7 PowerPC Processor

earth. The specific telemetry capability are subject to both the on-board transceiver selected as well as the type of ground stations employed for the mission.

\section{Communications}

The communications module provides the interface between the on-board processor and the RF transceiver. For ground testing this hardware can be as simple as an ethernet module that connects to wireless network components. Although the flight module must also have a port for ground testing, it connects to a transceiver that is compatible with satellite network hardware. The current RF transceiver selection is a novel low mass SGLS-signaling AFSCN-compatible unit designed by the Naval Research Laboratory (NRL). More detailed information may be obtained by consulting the NRL Naval Center for Space Technology specifications SSD-S-CM013 and SSD-SCM017.

\section{Image Acquisition and Processing}

The current digital frame grabber module is a COTS board designed to provide a high-performance image acquisition and data handling interface between the CompactPCI bus and high-speed digital cameras. It features 8 to 16 bit pixels, pixel clock rates of up to $20 \mathrm{Mhz}$, multiplexed operation for cameras sharing the video channel, Automated Imaging Association (AIA) digital camera compatibility, a Look Up Table (LUT) to allow real-time hardware functions such as thresholding, a $16 \mathrm{~K}$ by $32 \mathrm{bit}$ FIFO buffer to support DMA over the CompactPCI bus, and a Region of Interest (ROI) acquisition mode. For additional image processing capability, the next-generation frame buffer module will be a commercial DSP board with local storage and an add-on mezzanine designed for hardware-based image compression. 


\section{Vehicle Control}

The system interface module provides connection, control, and acquisition functions for the MicroSat guidance and navigation elements. The current module is an Industry Pack (IP) carrier board that uses serial IP modules to communicate with the IMU, GPS, and Ranging components. A digital I/O module controls the valve drivers. Operations that require physical connections outside the MicroSat are handled via the external interface module. This includes control of mechanisms used in docking and servicing mission phases such as robotic arms, grappling fixtures, and electrical connectors.

\section{Power Distribution System}

The current MicroSat ground test vehicles have used low cost rechargeable Ni-Cad cells. We plan to utilize Li-ion rechargeable batteries that are undergoing qualification for space under Air Force sponsored efforts. There are several advanced solar cell technologies that can be used for future flight vehicles. Dual-junction cells and concentrator arrays offer the ability to maximize power generation in very low mass and small areas. A specific choice will await future flight design efforts.

\section{Power Management}

The power management scheme uses the approach as described previously ${ }^{1}$. The power management controller monitors and controls the power to all of the spacecraft loads. It communicates with the system processor to provide the capability to make mode changes, carry out power down commands to various components, and monitor the power system condition. This link also provides a way for the controller to signal the system processor of power system alarms and to alert it to the need for imminent shutdown. The MicroSat will enter a powerdown mode autonomously by the system processor as a result of preprogrammed mission operations or as a result of detecting battery depletion via the master power distribution module. A second means is receipt and confirmation of a power-down command from the ground station.

\section{Software}

The MicroSat software development environment uses the VxWorks real-time operating system (RTOS). VxWorks is a commonly used, well-tested, RTOS that provides a rapid development environment for integration of new software modules. It is also portable among many processors. It has been used in space applications including JPL's Mars Pathfinder and the Clementine I spacecraft. Figure 8 shows the hierarchical organization of our mission software. Many software modules for GN\&C, imaging, target tracking, and other real-time operating codes can be adapted for the representative satellite mission.

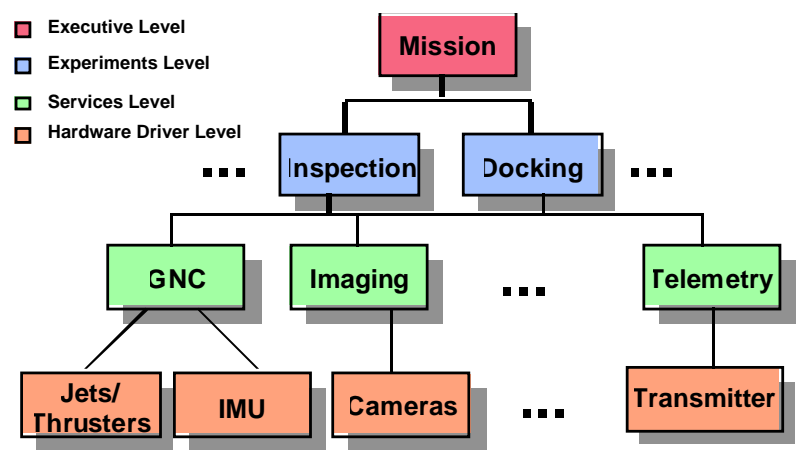

\section{VxWorks Operating System}

Figure 8 Hierarchical organization of mission software.

Most recently, we implemented the GN\&C code developed by Octant, Inc. for an Air Force micro-satellite mission, and validated their de-spin mode, inertial-attitude-hold mode and also the closed-loop divert (lateral-translation) operations on our indoor rail test vehicle. Fitting the Octant code into our development environment only took one programmer a couple of weeks.

\section{Integrated Testing}

Ground performance testing is the key to the success of a MicroSat mission. It is crucial to be able to repeatedly practice and test the integrated vehicle's ability to perform precision orientation and translational maneuvers. These tests should include maneuvers to achieve orbit matching and rendezvous, inspection and proximity-operations, docking, satellite servicing, and un-docking. Ideally, one would like to have a 6 Degrees-of-Freedom (DOF) test environment. However, in most cases a 5 DOF or 4 DOF environment is sufficient.

In order to support the testing of integrated MicroSats, LLNL has developed 4 DOF and 5 DOF dynamic air bearing ground testing facilities. The 4 DOF facility is an air rail with 3 degrees of rotational freedom and one degree of translational freedom. The 5DOF facility is an air table with 3 degrees of rotational freedom and two degrees of translational freedom. These facilities enable low cost repeatable end-to-end performance testing of integrated MicroSat testbed vehicles, and full-up performance acceptance testing of final flight hardware and software before launch.

During the Fall of 1998, an outdoor 40 meter air rail was set up to support the integrated "Hot Fire" testing of the 
latest generation of MicroSat test vehicle incorporating the self-pressurizing pumped propulsion system. A 40 meter air rail was used to provide long lateral thruster pulsing and large translations to evaluate vehicle stability during thruster firing. The large $(20 \mathrm{~N})$ liquid thrusters were mounted transversely to the vehicle long axis and to the sensor lineof-sight. Stable operation of the vehicle was demonstrated using the warm-gas ACS thrusters to maintain vehicle attitude throughout the run. These long baseline transverse maneuvers simulate some contemplated MicroSat inspection mission geometries. With the long rail a complex sequence of maneuvers were executed, including one run that transitioned from transverse thrusting to axial thrusting (using warm-gas thrusters aligned along the "long" vehicle axis). These maneuvers are contemplated for conditions where it is necessary to maintain propulsive acceleration along a line in space while pointing cameras at a fixed object, thus one needs to transition from one translational thruster to another while rotating the vehicle. The ability to track the target at a higher crossing velocity will tax the warm-gas attitude control system and therefore provide a higher assurance of the propulsion system performance. Figures 9 and 10 show the ETV-200 vehicle in close-up view and during testing respectively.

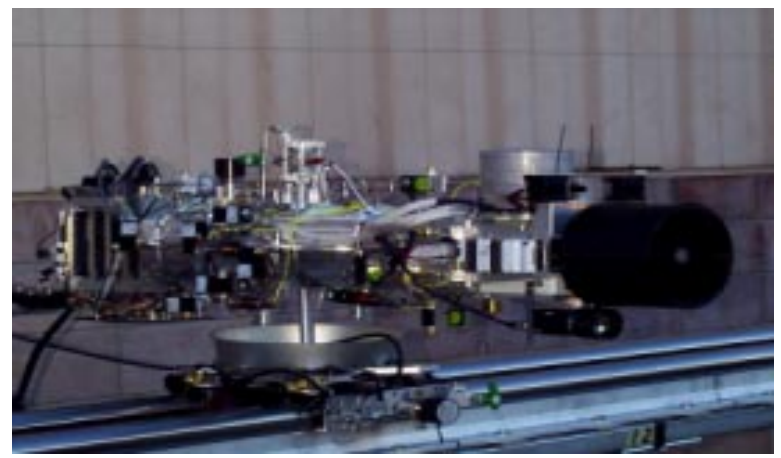

Figure 9 Warm gas ETV-200 vehicle in close-up view.

The purposes of the Hot Fire propulsion experiment are to first demonstrate the operation of the self-pressurizing micro-pumped $\mathrm{H}_{2} \mathrm{O}_{2}$ propulsion system design and then to demonstrate the precision maneuvering capability of the Engineering Testbed Vehicle equipped with this propulsion system. Finally, we need to measure and collect performance data on the propulsion system and vehicle maneuvers using the on-board control and data collection electronics and guidance and control software. The eventual goal is to provide a testbed vehicle that can experimentally verify MicroSat inspection and logistics mission maneuver requirements.

Figure 11 below describes a set of general maneuvers that simulate a potential MicroSat mission. Execution of a series of simple maneuvers are used to demonstrate the versatility

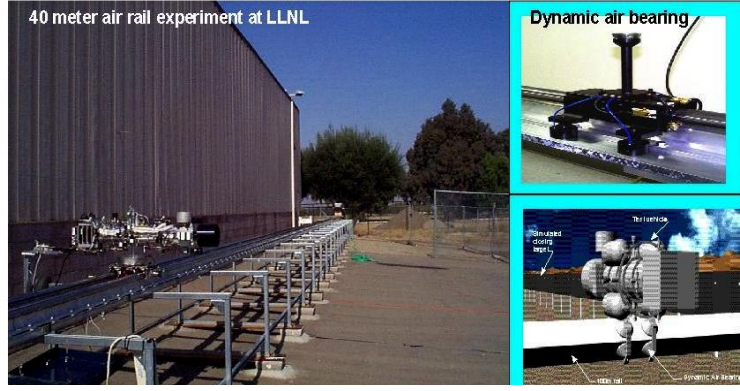

Figure 10 Warm gas DACS stabilized and propelled the vehicle during testing.

and utility of both the $\mathrm{H}_{2} \mathrm{O}_{2}$ transverse thrusters and the warm-gas ACS system. As shown in the figure, there are eleven executions ranging from demonstrating simple attitude control in pitch, roll, and yaw, to making both transverse and axial translations, and a simulated soft docking maneuver as the vehicle is propelled down the 130 foot air rail. On the return trip, the vehicle is demonstrating a maximum transverse $\Delta \mathrm{v}$ maneuver while maintaining vehicle attitude stability and control.

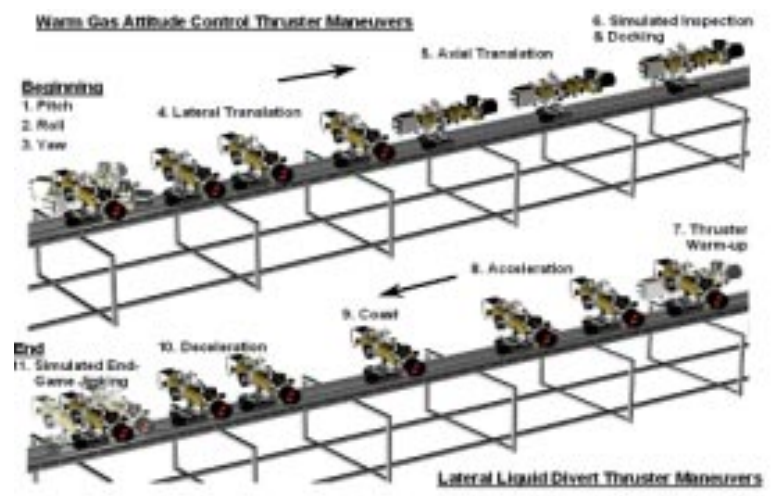

Figure 11 Maneuvering control experiment sequence on an air-rail.

Figure 12 shows the resulting roll, pitch, and yaw motions and the transverse thrusting sequence. Figure 13 shows the yaw ACS jet time histories. Note that an additional $360^{\circ}$ maneuver was added near the end at the $60 \mathrm{~s}$ mark to simulate a target search. The preliminary results of the experiment demonstrated that the new non-toxic selfpressurizing propulsion system is operational. The vehicle achieved a vehicle acceleration of $0.1 \mathrm{~g}$. Measurements of the pointing stability during the pure translational portions of the experiment (10 to 40 seconds) show that the pitch axis was held to a precision attitude pointing of 0.16 degrees.

American Institute of Aeronautics and Astronautics 

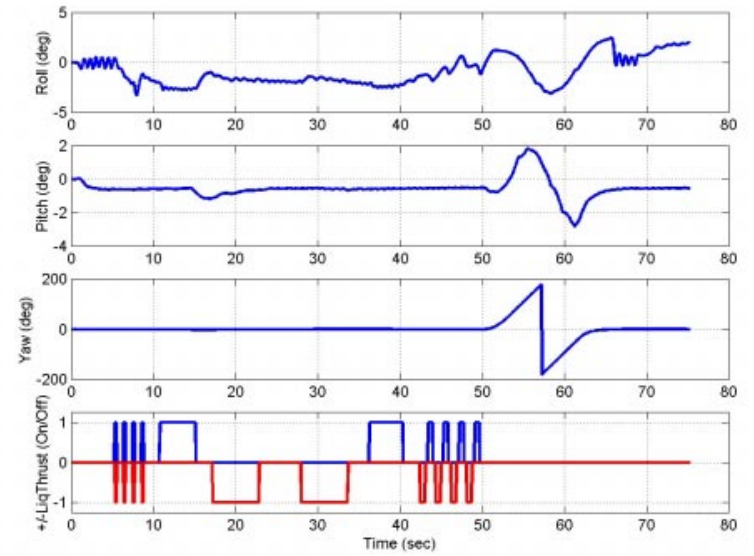

Figure 12 Hot fire experiment vehicle roll, pitch, yaw and transverse thrusting time histories.

Prior to the hot fire experiment, vehicle control software was developed and debugged on a cold gas prototype vehicle operating on an indoor $20 \mathrm{~m}$ air-rail as shown in Figure14. The air-rail flight software was validated using a 6-DoF simulation that predicted the vehicle performance during the experiment. The indoor facility was used to conduct Monte Carlo runs. Figure 15 shows the results of an experiment ${ }^{3}$ that is equivalent to a close fly-by inspection
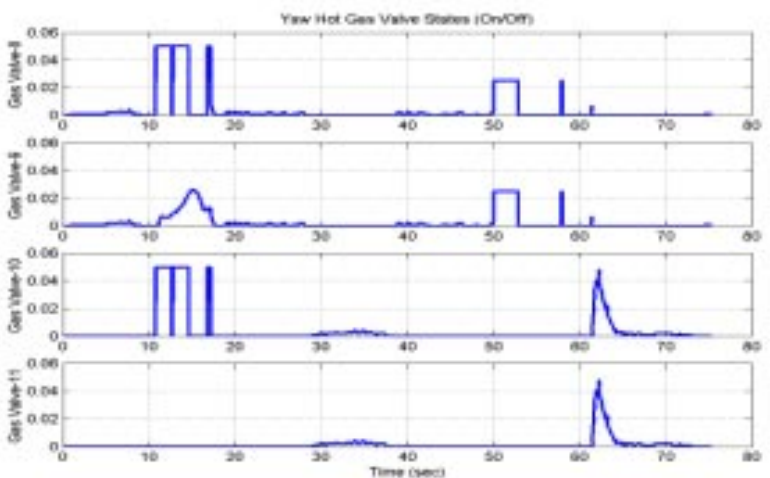

Figure 13 Hot fire experiment yaw ACS jet time histories.

mission of a target satellite at a very low closing velocity of $10 \mathrm{~m} / \mathrm{s}$. The mission is started at 100 meters from the target leaving a time to closest approach of $10 \mathrm{~s}$. This preliminary experiment did not include orbital effects and would simulate behaviors in very high orbits where the vehicle is essentially in an inertial frame. We are interested in estimating collision avoidance capabilities of the MicroSat by measuring the miss distance performance for different transverse acceleration capabilities for the vehicle. Note the close correlation between simulation and experimental results. As predicted smaller miss-distances were possible using larger transverse accelerations for the vehicle.

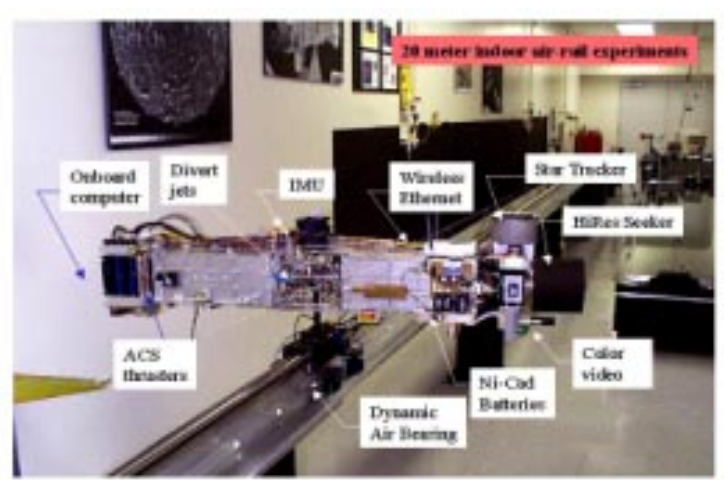

Figure 14 Prototype vehicle used for the AGILE experiments.

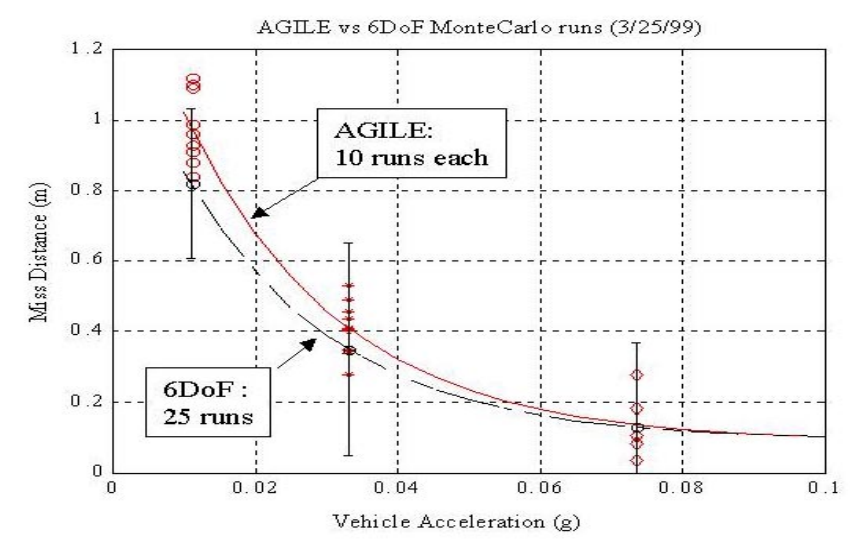

Figure 15 AGILE LOS rate prediction versus 6 DOF runs.

\section{Summary}

The MicroSat Technologies Program is continuing to develop more capable MicroSat ground test vehicles with new sensor, avionics and propulsion technologies. New ground test capabilities enable complex high fidelity performance testing this new class of agile MicroSats. Work is still in progress to develop advanced pump-fed propulsion systems that can directly feed liquid thrusters at high pressures, enabling reduced propellant tank masses and larger mass ratios resulting in MicroSats with multikilometer per second $\Delta \mathrm{v}$ capability. Coupling this propulsion capability with advanced integrated sensors, avionics and intelligent GN\&C software will enable these vehicles to autonomously perform complex space logistics missions. A series of docking experiments are planned to evaluate the various sensing technologies and GN\&C strategies necessary to enable a MicroSat to perform these maneuvers. 


\section{Acknowledgments}

The authors would like to thank the entire MicroSat Technologies Program Team including Don Antelman, Germaine Brassinga, Ed English, Lisa Hensel, Darron Nielsen, Gloria Purpura, Jeff Robinson, Bill Taylor and Dean Urone.

The research was part of the Microsat Technologies Program at LLNL, supported by the U.S. Air Force Research Laboratory. This work was sponsored by the U.S. Government and performed by the University of California Lawrence Livermore National Laboratory under Contract W-7405-Eng-48 with the U.S. Department of Energy.

\section{$\underline{\text { References }}$}

1. Ledebuhr, A.G., J.F. Kordas, et al, "Autonomous, Agile, Micro-Satellite Technology for Use in Low Earth Orbit Missions," SSC98-V-1, The $12^{\text {th }}$ Annual Utah State University Small Satellite Conference, 1998.

2. Whitehead, J.C., Dittman, M.D., and Ledebuhr, A.G., "Progress Toward Hydrogen Peroxide Micropropulsion", SSC99-XII-5, The $13^{\text {th }}$. AIAA/USU Conference on Small Satellites, 1999.

3. L.C. Ng, E. Breitfeller, A.G. Ledebuhr, and F.A. Handler, "Air-Bearing Guided Intercept and Line-of-sight Experiments," Presented at the First Biennial AIAA National Forum on Weapon System Effectiveness, April 68, 1999, Eglin AFB, Ft. Walton Beach, FL. 